\title{
Current and future development of the photoionization code Cloudy
}

\author{
P.A.M. van Hoof ${ }^{1}$, G.C. Van de Steene ${ }^{1}$, F. Guzmán ${ }^{2}$, \\ M. Dehghanian ${ }^{2}$, M. Chatzikos ${ }^{2}$ and G.J. Ferland ${ }^{2}$ \\ 1 Royal Observatory of Belgium, Ringlaan 3, B-1180 Brussels, Belgium \\ 2 Department of Physics and Astronomy, The University of Kentucky, \\ Lexington, $K Y$ 40506, USA
}

Received: July 31, 2019; Accepted: October 24, 2019

\begin{abstract}
The interstellar medium (ISM) plays a crucial role in the cycle of matter in every galaxy. The gas and dust that is present in the ISM is usually very far removed from (local) thermodynamic equilibrium, and in some cases may also not be in a steady-state equilibrium with its surroundings. The physics of this material is complex and you need a sophisticated numerical code to study it. For this purpose the open-source photoionization code Cloudy was created. It models the physical state of the gas and predicts the spectrum that it emits.

Cloudy is continually being developed to improve the treatment of the microphysical processes and the database of fundamental data that it uses. In this paper we will discuss how we are developing the code to improve our highdensity predictions by implementing better collisional-radiative models for all ions. We will also briefly discuss the experimental mode in Cloudy to model gas that is not in steady-state equilibrium and present a preliminary model of recombining gas in a planetary nebula that is on the cooling track. We finish with a short discussion of how we are speeding up the code by using parallelization.

Key words: plasmas - ISM: general - planetary nebulae: general
\end{abstract}

\section{Introduction}

The space between stars is filled with a very tenuous gas called the interstellar medium (ISM) which plays a crucial role in the evolution of every galaxy. This medium is usually irradiated by strongly diluted radiation fields and is therefore far removed from local thermodynamic equilibrium (LTE). In some cases it may also not be in a steady-state equilibrium with its surroundings. The material in the ISM is generally primarily heated by stellar light, but other energy sources could be shocks, magnetic reconnection, cosmic rays, radioactive decay, etc. The gas may be ionized, neutral, or molecular and usually also contains dust grains. The geometry of the gas is often intricate, making solving the radiative transfer equations difficult. As a result the physics of this material is complex and you need a sophisticated numerical code to model the spectrum emitted by this gas. 
For this purpose the open-source photoionization code Cloudy was created on 28 August 1978 in Cambridge, UK. The emphasis is on detailed treatment of microphysical processes, but it also needs simplifying assumptions, such as 1D spherical geometry ${ }^{1}$ with simplified radiative transfer, no treatment of shocks, only a very basic treatment of magnetic fields, and no inclusion of radioactive decay. It models the physical state of the gas and predicts the spectrum emitted by that gas. It is the only code that can make a self-consistent model of a photoionized region and the neutral and molecular regions beyond the ionization front. Such a code needs a vast amount of atomic and molecular data. Cloudy is continually being developed to improve the treatment of the microphysical processes and the database of fundamental data that it uses, with the aim of making the code suitable for the widest possible range of physical conditions. In this paper we will briefly discuss our ongoing efforts to improve the code. We will also discuss the experimental mode in Cloudy to model gas that is not in steady-state equilibrium and show results from a preliminary model of a recombining planetary nebula $(\mathrm{PN})$. In the final section we briefly discuss how we are speeding up the code using parallelization.

\section{The ionization equilibrium}

To meet our goal of modeling a wide range of physical conditions, Cloudy needs to be able to calculate the ionization balance over a wide range of densities. In very low-density gas, the ionization balance can be derived using the twolevel or coronal approximation. Here it is assumed that all the material is in the ground state, and hence you only need to consider two states: the ground state of the current and the next ion. This approximation is valid because the time between two interactions is very long, giving the ion enough time to relax into the ground state after an excitation or recombination event. At very high densities, the collisional rates will be very fast, driving the gas into LTE. This implies that the ionization balance can be derived from the Saha-Boltzmann equation. Both limits are very easy to model, however at intermediate densities neither limit is valid and a complex numerical collisional-radiative model (CRM) is needed to obtain accurate predictions for the ionization balance. This is shown in Fig. 1.

A CRM solution requires many excited states to be included in the model atom as the average time between two interactions is too short for the electron to reach the ground state and excitations or ionizations from excited states become important. In recent years a considerable amount of effort was dedicated to prepare Cloudy for this task. Here we will describe the most important aspects of this work.

\footnotetext{
${ }^{1}$ Christophe Morisset implemented pseudo-3D modeling based on Cloudy models using the pyCloudy package (Morisset, 2013).
} 


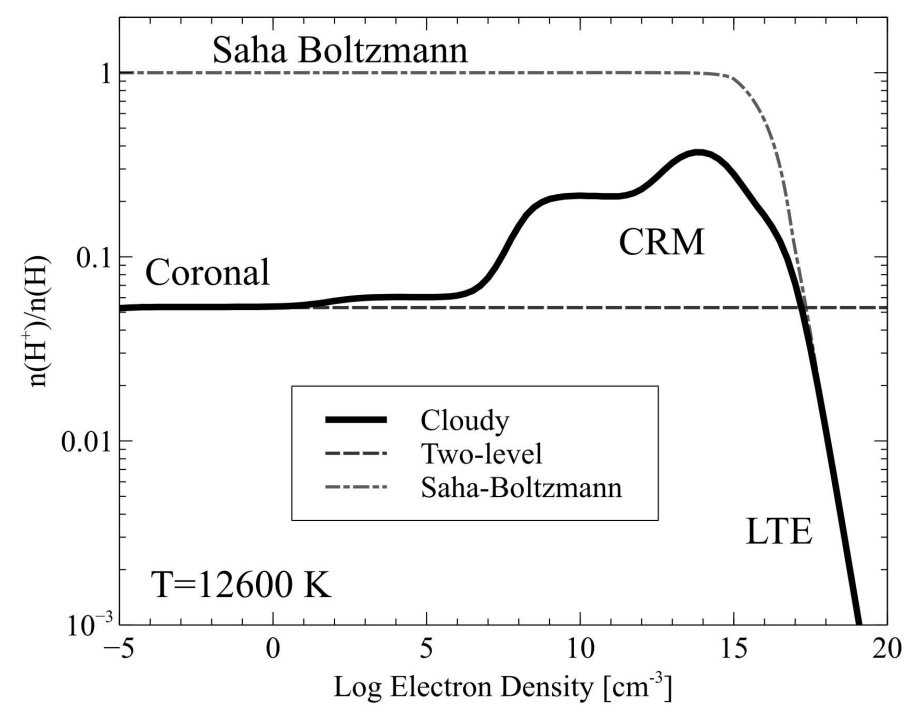

Figure 1. Ionization of hydrogen as a function of density. The solid line is the full numerical CRM solution, the dashed line is the ionization predicted by the two-level approximation, and the dashed-dotted line is the prediction of the Saha-Boltzmann equation. Figure taken from Ferland et al. (2017).

\subsection{H- and He-like ions}

For the H- and He-like iso-electronic sequences we use a custom approach for setting up the model atoms. This is done in such a way that the user can request an arbitrary number of levels. These can either be l-resolved or collapsed into states with the same principal quantum number $n$. Such an approach is possible by exploiting regularities in the atomic data resulting from the simple level structure of these ions. The original implementation of the He-like sequence is discussed in Porter et al. (2005); Bauman et al. (2005) and the H-like sequence in Luridiana et al. (2009). For the 2017 release of Cloudy we started a comprehensive review of these iso-electronic sequences, mainly focusing on the accuracy of the collisional data we use (Guzmán et al., 2016, 2017, 2019). This effort is still ongoing and will result in further updates in a future release.

The H- and He-like ions are unusual because the first excited level is much closer to the ionization threshold than to the ground state (see Fig. 2). This implies that in photoionized plasmas collisional excitation is generally unimportant, and the emission line spectra are produced by recombination. Recombination typically happens in highly excited Rydberg states, which are tightly coupled to the ionized continuum by collisional ionization and photoionization and their inverse processes. To make accurate predictions of the recombina- 


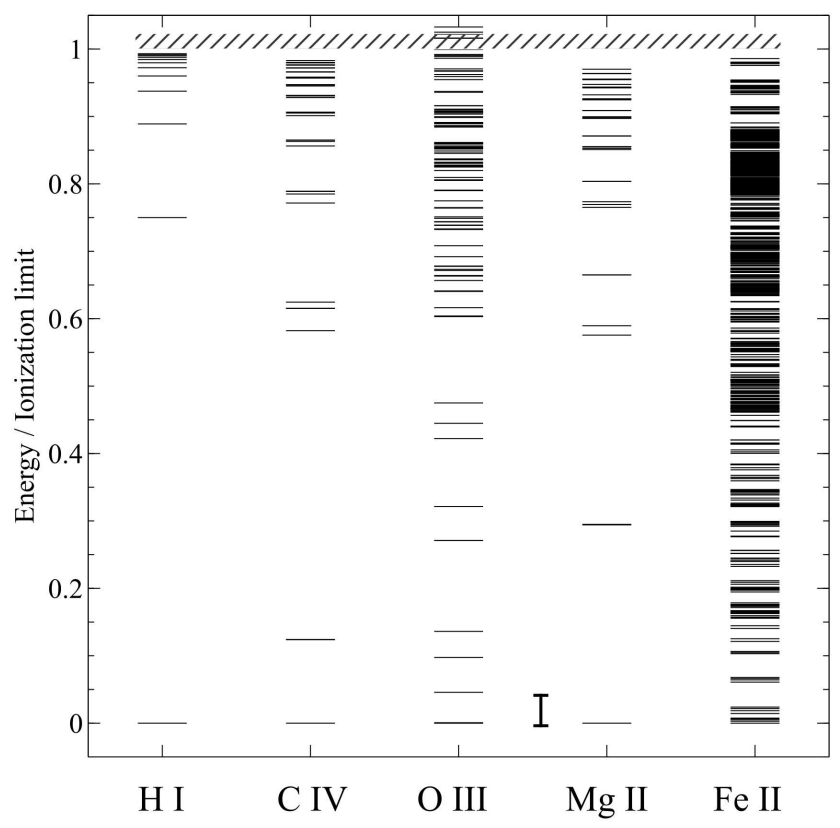

Figure 2. Experimental energy levels taken from NIST ASD (Kramida et al., 2014) for some species present in an ionized gas. The energies are given relative to the first ionization potential. Figure taken from Ferland et al. (2017).

tion spectrum, a very large model atom is needed. Cloudy is capable of setting up such a model atom, but the CPU and memory requirements are currently prohibitive. The reason for that is twofold. On the one hand, calculating the atomic data is too slow, while on the other hand solving the equations for the level populations takes too much time as every level is modeled explicitly. We will tackle the former problem by caching atomic data or moving them into data files (possibly in Stout format), rather than calculating them on the fly, and also by using better optimized algorithms. To alleviate the latter problem, we will implement the matrix condensation technique pioneered by Burgess \& Summers (1969) and Brocklehurst (1970). This technique is akin to the superlevel technique often used in stellar atmospheres, which allows a significant reduction in the number of levels that are modeled, thus significantly speeding up the LU decomposition needed to solve the level populations. This will allow us to routinely model large model atoms and improve the accuracy of our recombination line predictions. This is e.g. needed for the measurement of the primordial helium abundance. These improvements will be offered in a future release of Cloudy. 


\subsection{More complex ions}

Unlike H- and He-like ions, more complex ions have low-lying excited states. These can be collisionally excited, even in a photoionized plasma and thus can be an important source of cooling for the gas. Moreover, these ions can lack the regularities that $\mathrm{H}$ - and $\mathrm{He}$-like ions have, e.g., due to configuration interaction shifting levels out of position. Because of this, a very different approach is needed compared to what we described above. We will need to tabulate the atomic data that are needed to model the atom. The level energies will normally be taken from experiments, while the transition probabilities and collision strengths typically come from large-scale computer models.

With the 2013 release of Cloudy we started moving these atomic data into external data files (until then, the data for a relatively small number of important lines were hardwired into the code). Initially we adopted the Chianti database (Dere et al., 1997; Landi et al., 2012) but we quickly realized that the scope of this database is insufficient for us to depend on it exclusively (e.g., it contains insufficient data for low ionization stages). So we decided to develop our own Stout database for atomic and molecular data. This database was introduced in the 2013 release of Cloudy (Ferland et al., 2013), but was only used for a very limited number of ions. With the 2017 release of Cloudy we moved many more ions to the Stout database, vastly increasing the number of levels and lines that can be modeled with Cloudy. A more detailed discussion of these points can be found in Ferland et al. (2017).

This is the first step towards full CRM treatment of these ions. However, more work is needed. Many of the entries in the Stout database are what we call "baseline" models. These contain level energies and transitions probabilities taken from NIST ASD (Kramida et al., 2014) but no collisional data. If for a given transition the collisional data are missing, Cloudy will use the g-bar approximation (Burgess \& Tully, 1992) which is now widely viewed as unreliable, but is still better than having no data at all. To improve this situation, we have started a project to update the baseline models using the latest level energies and transition probabilities from NIST ASD and combining those with electron impact collisional data from the OPEN-ADAS database ${ }^{2}$. This should vastly improve the quality of the predictions for these model atoms.

Solving the level populations and the ionization balance are however still not coupled in the sense that ionization from excited states is not considered for these complex ions (with only a handful of exceptions that are currently hardwired into the code). We aim to improve this situation in collaboration with the University of Cambridge by including both collisional ionization and photoionization processes from metastable states. All these improvements will be offered in a future release of Cloudy.

${ }^{2}$ http://open.adas.ac.uk/ 


\section{Time-dependent modeling}

For quite some time Cloudy has been able to model time-steady non-equilibrium dynamical flows (Henney et al., 2005, 2007). This capability was later extended to modeling time-dependent non-equilibrium conditions in the gas (Chatzikos et al., 2015). This feature is still considered experimental. Here we apply this method to the problem of a recombining $\mathrm{PN}$.

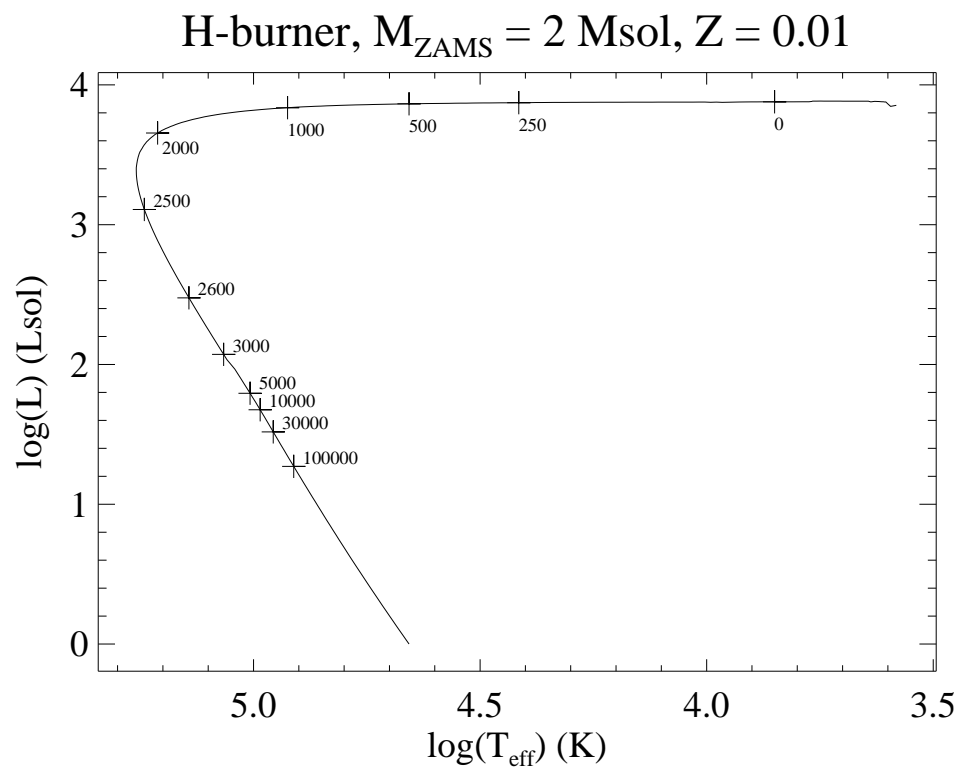

Figure 3. Evolutionary track from Miller Bertolami (2016). Ages in years since the star left the AGB are marked on the track.

When an intermediate-mass star nears the end of its life, it will experience thermal pulses during the asymptotic giant branch (AGB) part of its evolution. At this time the energy generation is via hydrogen shell burning, punctuated at regular intervals $\left(10^{4}-10^{5} \mathrm{yr}\right)$ by helium flashes followed by short phases of quiescent helium burning. During these flashes, the remaining envelope of the star is removed in a superwind. When the envelope is nearly depleted, the star starts to heat up and ionizes the material that was ejected during the AGB phase, forming a new PN. Eventually the hydrogen burning shell runs out of fuel and the nuclear burning will shut down. At this time the luminosity of the star will quickly go down while it also will start to cool, eventually turning the star into a new white dwarf. This part of the evolution is called the cooling track. 
When the star is on the cooling track, the PN will receive increasingly less ionizing photons. This implies that the outer parts of the $\mathrm{PN}$ can no longer be ionized and will start to recombine (e.g., van Hoof et al., 2000). This is a nonequilibrium process that we can model with Cloudy. Here we present preliminary results. To have a realistic evolution of the luminosity as a function of time, we use the $2 \mathrm{M}_{\odot}$ hydrogen-burning evolutionary track from Miller Bertolami (2016) with $Z=0.01$. This track is shown in Fig. 3 .

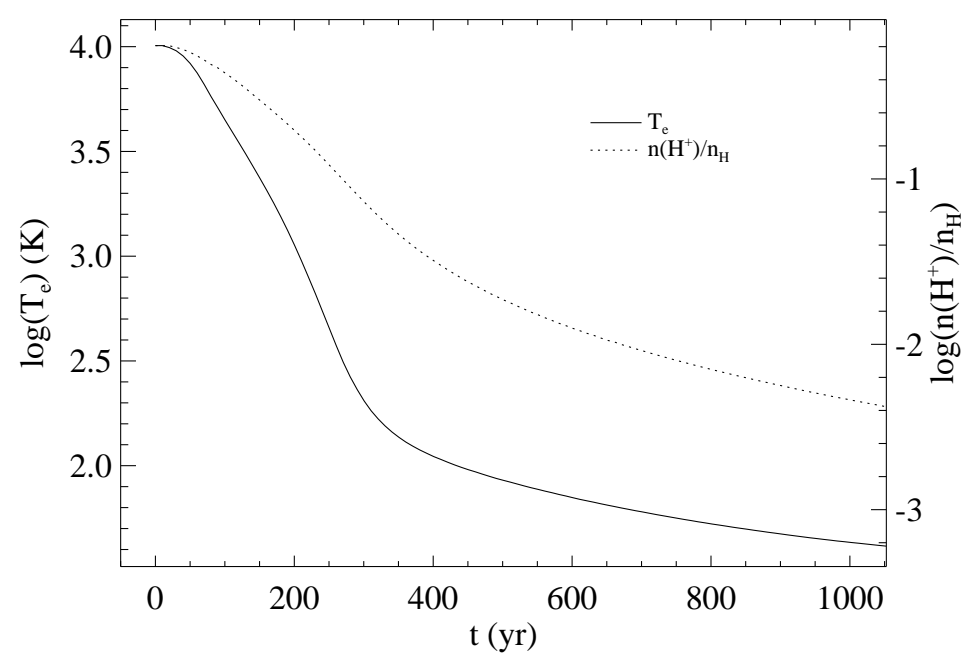

Figure 4. The evolution of the electron temperature and hydrogen ionization fraction as a function of time at a radius of $4 \times 10^{15} \mathrm{~m}$.

We will start the Cloudy model when the star is at the maximum temperature, $T_{\text {eff }}=181.5 \mathrm{kK}$. This point is reached after $2404 \mathrm{yr}$. It is currently not possible to vary the stellar temperature, so we assume that it stays constant at this value during the evolution. We model the spectrum of the star using the H-Ca grid of atmosphere models by Rauch (1997). The evolution of the luminosity as a function of time will be given by the evolutionary track, starting at a luminosity of $2455 \mathrm{~L}_{\odot}$. The gas is assumed to have a constant hydrogen density of $1000 \mathrm{~cm}^{-3}$, a reasonable value for an evolved PN. The inner radius is set at $1.135 \times 10^{15} \mathrm{~m}$, which is the distance the gas would have traveled in $2404 \mathrm{yr}$ assuming a canonical expansion velocity of $15 \mathrm{~km} \mathrm{~s}^{-1}$. The outer radius is set at $5.395 \times 10^{15} \mathrm{~m}$, chosen to coincide with the ionization front at the start of the simulation. This implies an ionized mass of $0.75 \mathrm{M}_{\odot}$. The gas is assumed to contain graphite dust (Martin \& Rouleau, 1991).

The results of the model are shown in Figs. 4 and 5. The first shows that the electron temperature drops very quickly: the decrease from $10116 \mathrm{~K}$ to $1000 \mathrm{~K}$ 


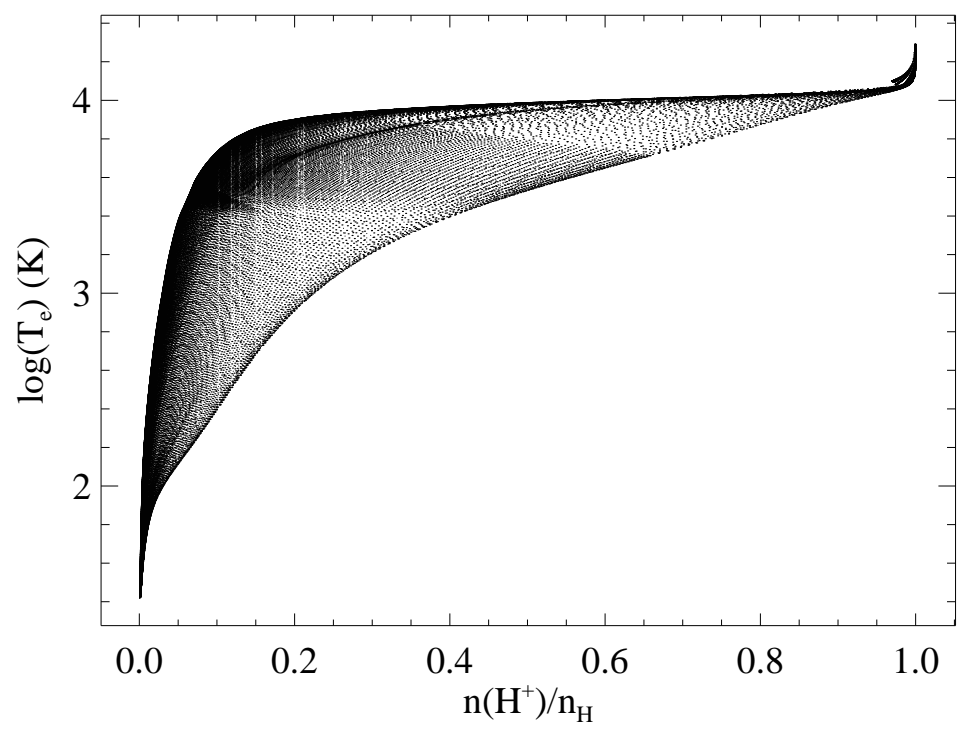

Figure 5. The electron temperature as a function of hydrogen ionization fraction is shown for every depth point of every time step of the model.

happens in less than $210 \mathrm{yr}$, while the hydrogen ionization fraction lags behind. When the electron temperature reaches $1000 \mathrm{~K}$, hydrogen is still $\approx 18 \%$ ionized. This can be seen even more clearly in Fig. 5 where we show the electron temperature as a function of the degree of ionization for all depths and every time step. From this it becomes clear that in a recombining PN it is possible to observe partially ionized gas at remarkably low temperatures: at $50 \%$ ionization, the electron temperature can be as low as $3410 \mathrm{~K}$, at $20 \%$ ionization, as low as $815 \mathrm{~K}$, and at $10 \%$ ionization as low as $250 \mathrm{~K}$ !

Roughly similar behavior can be observed for $\mathrm{O}^{+}$, while $\mathrm{He}^{+}$and $\mathrm{N}^{+}$also follow this trend, but with less extreme temperature lows. On the other hand, ions with a higher ionization potential, such as $\mathrm{He}^{2+}, \mathrm{C}^{2+}, \mathrm{N}^{2+}$, and $\mathrm{O}^{2+}$, do not show this behavior. Their recombination timescales are much shorter and they will have recombined before the gas has had the chance to cool by a significant amount. So in a recombining PN, you can have very cold gas that still has appreciable amounts of $\mathrm{H}^{+}, \mathrm{He}^{+}, \mathrm{N}^{+}$, and $\mathrm{O}^{+}$, but not many other ions $\left(\mathrm{C}^{+}\right.$is also present, but that has a lower ionization potential and is still actively photoionized). This gas will still emit radiation due to the progressing recombination of these ions. These conclusions qualitatively agree with the earlier studies of Ferland \& Truran (1981) who were modeling a nova shell and Binette \& Robinson (1987) who were modeling an active galaxy. 
It is well known that evolved PNe, such as NGC 6720 (the Ring nebula) and NGC 7293 (the Helix nebula), can have a large number of dense, molecular knots embedded in the ionized gas (Matsuura et al., 2009). The origin of these knots is still debated. In O'Dell et al. (2007); van Hoof et al. (2010); Van de Steene et al. (2015) it has been proposed that these knots are not remnants of an earlier phase of evolution, but form in recombining gas when the star is on the cooling track. This leaves only one to two thousand years to form the knots in the case of NGC 6720 (O'Dell et al., 2007). They would form as a result of an instability. When the recombining gas is cold, its internal pressure is low, making it susceptible to compression, e.g., by recombination radiation. The preliminary models presented in this paper show that this proposal could be viable. The recombining gas indeed cools on a very short timescale, but stays partially ionized, so that it still emits recombination radiation. This agrees with the assumptions made in van Hoof et al. (2010).

\section{Parallelization}

Modeling large model atoms as well as doing time-dependent modeling require a lot of work to be done by the CPU. To ease that burden, it is imperative that the code makes efficient use of the hardware resources that are available. This can be improved either through using SIMD (Single Instruction Multiple Data) instructions (also called code vectorization), or by using multiple cores in parallel since modern computers have more and more cores available.

In recent years an effort has been made to vectorize Cloudy. One way of achieving this is to simplify loops such that the compiler can recognize the potential to use SIMD instructions and generate these automatically while compiling. For this the loop needs to carry out only one or two simple tasks, otherwise the compiler will quickly be overwhelmed by the complexity and compile it using scalar instructions. So to achieve automatic vectorization, it may be beneficial to split one complex loop into multiple simpler ones. This task has been carried out in CPU-critical parts of the code.

In addition, we also created code primitives that were explicitly written to use SIMD instructions. These include routines to carry out reduction loops and vectorized transcendental functions (which apply transcendental functions to an array of arguments). Using code vectorization resulted in a moderate speedup of the code (roughly 20\%). There likely is a potential to find further optimizations in the future. An added benefit is that any code that can be vectorized automatically by the compiler can also be parallelized automatically by the compiler using OpenMP.

Using multiple cores through parallelization promises more substantial time savings, but is also more complex to implement. In the past we have implemented parallelization at the highest level by calculating different models on different cores. This method is highly efficient, but can only be used in grid or optimizer 
runs. For a single model different methods are needed. You can use parallelization on a low level by parallelizing individual loops as was already discussed above. This approach also opens the possibility of using graphics processors (though one drawback is that these are largely optimized for single-precision floating point math, while Cloudy often uses double-precision variables). Another possibility is mid-level parallelization, e.g. by solving the level populations of different ions on different cores, or by solving different grain size bins on different cores. These are ideas that still need to be tested and implemented in the future.

\section{Concluding remarks}

The interplay of physical processes in photoionized and photo-dissociation regions is very complex. Modeling these processes requires a large-scale code, and maintaining this requires a continuous effort. This includes adding the latest atomic and molecular data sets, implementing new and improved theory describing the physical processes, implementing faster and better numerical methods, and of course fixing bugs. In this paper we have highlighted a couple of the projects that we have recently undertaken, are in the process of implementing, or are planning to start in the future. These will allow Cloudy to make more accurate predictions. They will also make the code faster and more versatile. We hope that these efforts will improve the usefulness of Cloudy for our users.

\section{References}

Bauman, R. P., Porter, R. L., Ferland, G. J., \& MacAdam, K. B., J-Resolved He I Emission Predictions in the Low-Density Limit. 2005, Astrophys. J., 628, 541, DOI: $10.1086 / 430665$

Binette, L. \& Robinson, A., Fossil nebulae in the context of active galaxies. I. Time evolution of a single cloud. 1987, Astron. Astrophys., 177, 11

Brocklehurst, M., Level populations of hydrogen in gaseous nebulae. 1970, Mon. Not. R. Astron. Soc., 148, 417, DOI: 10.1093/mnras/148.4.417

Burgess, A. \& Summers, H. P., The Effects of Electron and Radiation Density on Dielectronic Recombination. 1969, Astrophys. J., 157, 1007, DOI: $10.1086 / 150131$

Burgess, A. \& Tully, J. A., On the Analysis of Collision Strengths and Rate Coefficients. 1992, Astron. Astrophys., 254, 436

Chatzikos, M., Williams, R. J. R., Ferland, G. J., et al., Implications of coronal line emission in NGC 4696*. 2015, Mon. Not. R. Astron. Soc., 446, 1234, DOI: $10.1093 / \mathrm{mnras} / \mathrm{stu} 2173$ 
Dere, K. P., Landi, E., Mason, H. E., Monsignori Fossi, B. C., \& Young, P. R., CHIANTI - an atomic database for emission lines. 1997, Astron. Astrophys., Suppl., 125, 149, DOI: 10.1051/aas:1997368

Ferland, G. J., Chatzikos, M., Guzmán, F., et al., The 2017 Release of Cloudy. 2017, Revista Mexicana de Astronomía y Astrofísica, 53, 385

Ferland, G. J., Porter, R. L., van Hoof, P. A. M., et al., The 2013 Release of Cloudy. 2013, Revista Mexicana de Astronomía y Astrofísica, 49, 137

Ferland, G. J. \& Truran, J. W., An X-ray model for the nebula of nova DQ HER 1934. 1981, Astrophys. J., 244, 1022, DOI: 10.1086/158773

Guzmán, F., Badnell, N. R., Williams, R. J. R., et al., H, He-like recombination spectra - I. l-changing collisions for hydrogen. 2016, Mon. Not. R. Astron. Soc., 459, 3498, DOI: 10.1093/mnras/stw893

Guzmán, F., Badnell, N. R., Williams, R. J. R., et al., H-, He-like recombination spectra - II. l-changing collisions for He Rydberg states. 2017, Mon. Not. R. Astron. Soc., 464, 312, DOI: 10.1093/mnras/stw2304

Guzmán, F., Chatzikos, M., van Hoof, P. A. M., et al., H-, He-like recombination spectra - III. n-changing collisions in highly excited Rydberg states and their impact on the radio, IR, and optical recombination lines. 2019, Mon. Not. $R$. Astron. Soc., 486, 1003, DOI: 10.1093/mnras/stz857

Henney, W. J., Arthur, S. J., Williams, R. J. R., \& Ferland , G. J., SelfConsistent Dynamic Models of Steady Ionization Fronts. I. Weak-D and Weak-R Fronts. 2005, Astrophys. J., 621, 328, DOI: 10.1086/427491

Henney, W. J., Williams, R. J. R., Ferland, G. J., Shaw, G., \& O’Dell, C. R., Merged Ionization/Dissociation Fronts in Planetary Nebulae. 2007, Astrophys. J., Lett., 671, L137, DOI: 10.1086/525023

Kramida, A., Yu. Ralchenko, Reader, J., \& and NIST ASD Team. 2014, NIST Atomic Spectra Database (ver. 5.2), [Online]. Available: https://physics.nist.gov/asd [2014, November 28]. National Institute of Standards and Technology, Gaithersburg, MD.

Landi, E., Del Zanna, G., Young, P. R., Dere, K. P., \& Mason, H. E., CHIANTI-An Atomic Database for Emission Lines. XII. Version 7 of the Database. 2012, Astrophys. J., 744, 99, DOI: 10.1088/0004-637X/744/2/99

Luridiana, V., Simón-Díaz, S., Cerviño, M., et al., Fluorescent Excitation of Balmer Lines in Gaseous Nebulae: Case D. 2009, Astrophys. J., 691, 1712, DOI: $10.1088 / 0004-637 X / 691 / 2 / 1712$

Martin, P. G. \& Rouleau, F., Extreme Ultraviolet Opacity with Interstellar Dust. 1991, in Extreme Ultraviolet Astronomy, ed. R. F. Malina \& S. Bowyer, 341 
Matsuura, M., Speck, A. K., McHunu, B. M., et al., A "Firework" of $\mathrm{H}_{2}$ Knots in the Planetary Nebula NGC 7293 (The Helix Nebula). 2009, Astrophys. J., 700, 1067, DOI: 10.1088/0004-637X/700/2/1067

Miller Bertolami, M. M., New models for the evolution of post-asymptotic giant branch stars and central stars of planetary nebulae. 2016, Astron. Astrophys., 588, A25, DOI: 10.1051/0004-6361/201526577

Morisset, C. 2013, pyCloudy: Tools to manage astronomical Cloudy photoionization code, Astrophysics Source Code Library

O’Dell, C. R., Sabbadin, F., \& Henney, W. J., The Three-Dimensional Ionization Structure and Evolution of NGC 6720, The Ring Nebula. 2007, Astron. J., 134, 1679, DOI: 10.1086/521823

Porter, R. L., Bauman, R. P., Ferland, G. J., \& MacAdam, K. B., Theoretical He I Emissivities in the Case B Approximation. 2005, Astrophys. J., Lett., 622, L73, DOI: 10.1086/429370

Rauch, T., Implication of light metals (Li-Ca) on NLTE model atmospheres of compact hot stars. 1997, Astron. Astrophys., 320, 237

Van de Steene, G. C., van Hoof, P. A. M., Exter, K. M., et al., Herschel imaging of the dust in the Helix nebula (NGC 7293). 2015, Astron. Astrophys., 574, A134, DOI: 10.1051/0004-6361/201424189

van Hoof, P. A. M., van de Steene, G. C., Barlow, M. J., et al., Herschel images of NGC 6720: $\mathrm{H}_{2}$ formation on dust grains. 2010, Astron. Astrophys., 518, L137, DOI: 10.1051/0004-6361/201014590

van Hoof, P. A. M., Van de Steene, G. C., Beintema, D. A., et al., Properties of Dust Grains in Planetary Nebulae. I. The Ionized Region of NGC 6445. 2000, Astrophys. J., 532, 384, DOI: 10.1086/308536 